\title{
EFFECT OF DOPING CONCENTRATION ON THE OPTICAL PROPERTIES OF INDIUM-DOPED GALLIUM ARSENIDE THIN FILMS
}

\author{
MD. SAIFUL ISLAM, CHITRA DAS ${ }^{* 1}$, MEHNAZ SHARMIN ${ }^{2}$, KAZI MD. AMZAD \\ HUSSAIN $^{3}$ AND SHAMIMA CHOUDHURY
}

Department of Physics, University of Dhaka, Dhaka, Bangladesh

\begin{abstract}
Effects of indium doping (concentration 0.2, 0.3 and $0.4 \%$ ) on the optical properties of GaAs thin films were studied. Thin films of $600 \mathrm{~nm}$ were grown onto chemically and ultrasonically cleaned glass substrate by thermal evaporation method in high vacuum $\left(\sim 10^{-4} \mathrm{~Pa}\right)$ at $50^{\circ} \mathrm{C}$ fixed substrate temperature. The samples were annealed for 15 minutes at a fixed temperature of $200^{\circ} \mathrm{C}$. The thicknesses of films were being measured in situ by a quartz crystal thickness monitor during deposition. The transmittance and reflectance data were found using UV-VIS-NIR spectrophotometer in the photon wavelength range of $310 \sim 2500 \mathrm{~nm}$. These data were utilized to compute the absorption coefficient, refractive index, extinction co-efficient and band gap energy of the studied films. Here transmittance was found 78 for $0.2 \%$ indium doping concentration. The band gap energy decreased with the increase of doping concentration.
\end{abstract}

Key words: Doping concentration, Optical properties, GaAs thin films

\section{INTRODUCTION}

Today, III - V compound semiconductors are showcasing advanced performances in electronics and optoelectronics areas such as diode laser, light emitting diodes, photo detectors, electro-optic modulators and many more. GaAs which is a III - V compound semiconductor of zinc-blende structure has been focused for its potentially intrinsic advantages. The direct band gap of GaAs makes it an important candidate in the fields of manufacturing light emitting diodes and semiconductor lasers.

There are huge aspects in the study of ternary alloy semiconductor like $\operatorname{In}_{\mathrm{x}} \mathrm{Ga}_{1-\mathrm{x}} \mathrm{As}$ as it has a specific band structure. The purpose of using group III arsenide alloys is to obtain a material which consumes minimum power with maximum brightness (Srivani et al. 2014).

Doped or undoped GaAs can be prepared by many methods. Molecular beam epitaxy method was used for optical absorption and photoconductivity measurement of $50 \mathrm{~nm}$

*Corresponding author: <dchitra20@yahoo.com>.

${ }^{1}$ Department of Mathematics \& Natural Sciences, BRAC University, Dhaka, Bangladesh.

${ }^{2}$ Department of Physics, Bangladesh University of Engineering \& Technology, Dhaka, Bangladesh.

${ }^{3}$ Experimental Physics Division, Atomic Energy Centre, Dhaka, Bangladesh. 
ultra-thin films of single crystal GaAs (Halliday et al. 1995). Electrical conductivity and temperature dependence on GaAs thin films deposited by $\mathrm{RF}$ sputtering were measured (Tsuji et al. 1992). Thermal evaporation method was used for studying the effect of rate of deposition on the optical parameters of GaAs thin films (Majeed 2011). Biswas et al. (2016) studied structural and optical characterization of $\mathrm{Mg}$-doped $\mathrm{ZnO}$ thin films deposited by spray pyrolysis method. Das et al. (2013) prepared n-type GaAs thin films by vacuum evaporation methods and studied their optical properties. Optical and transport properties of p-type GaAs were also investigated (Sharmin et al. 2012). Hydrogen-doped GaAs thin films were made by RF magnetron sputtering technique and their structural, optical and electrical properties were studied (Yan-Ping et al. 2008). Optical properties of undoped and indium-doped tin oxide thin films were studied (Chowdhury et al. 2011). Research in physical properties of III - V arsenide ternary semiconductor alloys were done (Srivani et al. 2014).

The present work reports the effect of various indium doping concentration on the optical properties of vacuum evaporated thin films of GaAs via transmittance and reflectance measurements. Optical parameters such as absorption coefficient, refractive index, extinction co-efficient were determined. Optical process or absorption method was implied to determine the band gap energy.

\section{MATERIALS AND METHODS}

Thin films of indium-doped GaAs were prepared using a vacuum evaporation unit type Edwards 306A, UK in vacuum $\left(\sim 10^{-4} \mathrm{~Pa}\right)$ onto chemically and ultrasonically cleaned glass substrates by thermal evaporation method. The substrate temperature was fixed at $50^{\circ} \mathrm{C}$. The films were then thermally annealed in situ at the temperature of $200^{\circ} \mathrm{C}$ for 15 minutes. The thickness of the films were $600 \mathrm{~nm}$. The evaporation rate for GaAs was $0.2 \mathrm{~nm} / \mathrm{sec}$ which was measured in situ by the FTM5 quartz crystal thickness monitor (Edwards, UK). The concentration of indium doping was $0.2,0.3$ and $0.4 \%$.

The variations of transmittance and absolute specular reflectance of the films with wavelength of light incident on them were measured using a dual beam UV-VIS-NIR recording spectrophotometer (Shimadzu, UV-3100, Japan) in the photon wavelength range of 300 to $2500 \mathrm{~nm}$. Light signals coming from the samples were detected by an integrating sphere. The thickness of the composite films was checked using an infrared interference method with the spectrophotometer, which depends on the reflectance characteristics of the films. In this method the thickness of a film is given by

$$
d=\frac{\Delta m}{2 \sqrt{n_{1}^{2}-\sin ^{2} \theta}} \frac{1}{\left(1 / \lambda_{1}\right)-\left(1 / \lambda_{2}\right)}
$$


(1)

where, $n_{1}$ is the refractive index of the film, $\theta$ is the incident angle of light to the sample, $\lambda_{1}$ and $\lambda_{2}$ are the peak or valley wavelengths in the reflectance spectrum and $\Delta m$ is the number of peaks or valleys between $\lambda_{1}$ and $\lambda_{2}$, where $\lambda_{2}>\lambda_{1}$.

For transmittance $(T \%)$ at normal incidence and reflectance $(R \%)$ at near-normal incidence of light on the films, expressions for the multiple reflected systems have been given by Heavens (1995). Tomlin (1968) simplified these expressions for absorbing films on non-absorbing substrates and expressed as

$$
\begin{aligned}
& \frac{1+R}{T}=\frac{1}{4 n_{2}\left(n_{1}^{2}+k_{1}^{2}\right)}\left[\begin{array}{l}
\left(1+n_{1}^{2}+k_{1}^{2}\right)\left\{\left(n_{1}^{2}+n_{2}^{2}+k_{1}^{2}\right) \cosh 2 \alpha_{1}+2 n_{1} n_{2} \sinh 2 \alpha_{1}\right\} \\
+\left(1-n_{1}^{2}-k_{1}^{2}\right)\left\{\left(n_{1}^{2}-n_{2}^{2}+k_{1}^{2}\right) \cosh 2 \gamma_{1}+2 n_{2} k_{1} \sin 2 \gamma_{1}\right\}
\end{array}\right] \\
& \frac{1-R}{T}=\frac{1}{2 n_{2}\left(n_{1}^{2}+k_{1}^{2}\right)}\left[\begin{array}{l}
n_{1}\left(\left(n_{1}^{2}+n_{2}^{2}+k_{1}^{2}\right) \sinh 2 \alpha_{1}+2 n_{1} n_{2} \cosh 2 \alpha_{1}\right\} \\
+k_{1}\left\{\left(n_{1}^{2}-n_{2}^{2}+k_{1}^{2}\right) \sinh 2 \gamma_{1}+2 n_{2} k_{1} \cos 2 \gamma_{1}\right\}
\end{array}\right]
\end{aligned}
$$

where $n_{1}$ and $n_{2}$ are refractive indices of the film arztritutrate respec2innlyd $k_{1}$ is the extinction co-efficient of the film, $n_{2}=1.45, a_{1}=\frac{\lambda}{\lambda}$ and $\gamma_{1}=\frac{\lambda}{\lambda}$, where $\lambda$ is the wavelength of light and $d$ is the thickness of the film. Equations (1) and (2) are solved for $k_{1}$ and $n_{1}$ utilizing a computerized iteration process. The absorption coefficient, $\alpha$ is then calculated using $a=4 \pi k / \lambda$.

The optical band gap $E_{g}$ can be estimated from the following relation which is known as the Tuac plot (Tuac 1974):

$$
a h v=A\left(h v-E_{g}\right)^{n}
$$

where, $\mathrm{A}$ is a constant, $v$ is the transition frequency and the exponent $\mathrm{n}$ characterizes the nature of band transition. $\mathrm{n}=1 / 2$ and $3 / 2$ corresponds to indirect allowed and indirect forbidden transitions, respectively.

\section{RESULTS AND DISCUSSION}

Fig. 1 shows the optical transmittance spectra of indium doped GaAs thin films in the visible range. Transmittance shows better result approximately 78 for $0.2 \%$ indium concentration. Here increase of doping concentration reduces transmittance and shifts the peak of transmittance spectrum toward the higher wavelength. Decrease in transmittance may occur due to increase of particle size because of the progression of indium in the GaAs thin films (Salina et al. 2012). The shifting of peaks is also due to structural improvement i.e., increase of crystalline grain size (Balkanski and Wallis 2000). 


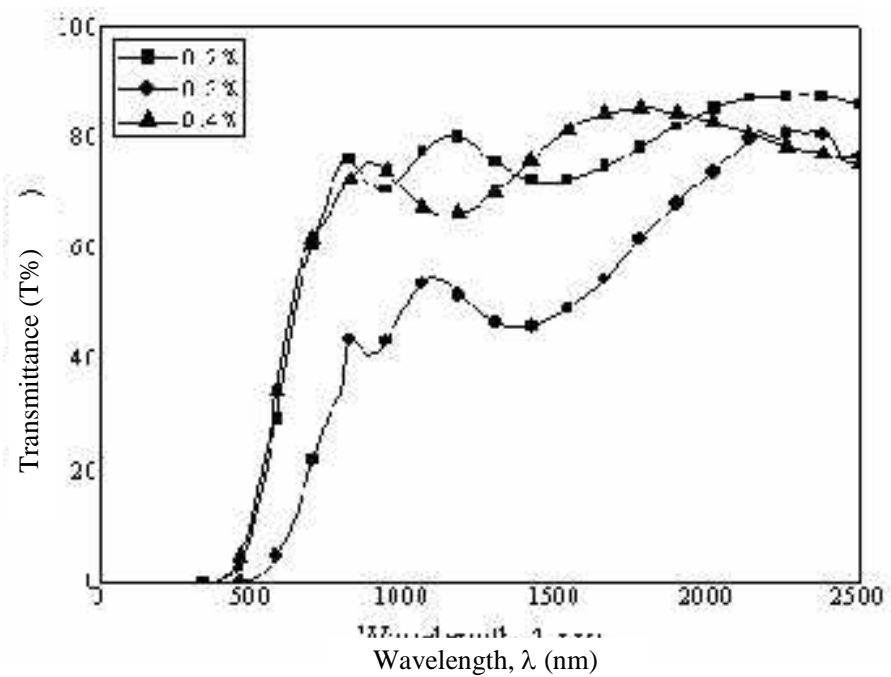

Fig. 1. The transmittance spectra at different indium concentration for $600 \mathrm{~nm}$ GaAs thin film.

The reflectance spectra have been presented in Fig. 2. Interference pattern with distinct rise and fall is observed in the reflectance spectra of the films.

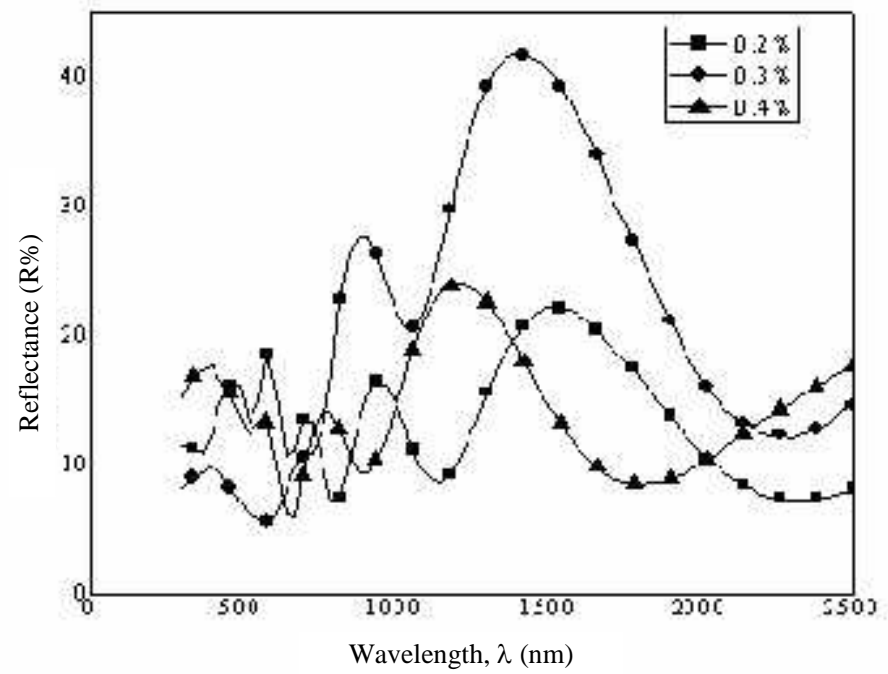

Fig. 2. The reflectance spectrum at different indium concentration for $600 \mathrm{~nm}$ GaAs thin film.

The behavior of absorbance spectra is shown in Fig. 3. It is observed that in the shorter wavelength the absorption co-efficient exhibits higher values $\left(>10^{4}\right)$. These higher values means there is a large probability of the allowed direct transition which agrees with other workers (Mott and Davis 1979). The absorption co-efficient decreases with increasing wavelength because of inverse relation between transmission and absorption (Majeed 2011) and remains consistent at higher wavelengths. The 
semiconductor alloys with higher value of absorption co-efficient can be used for photovoltaic devices (Srivani et al. 2014).

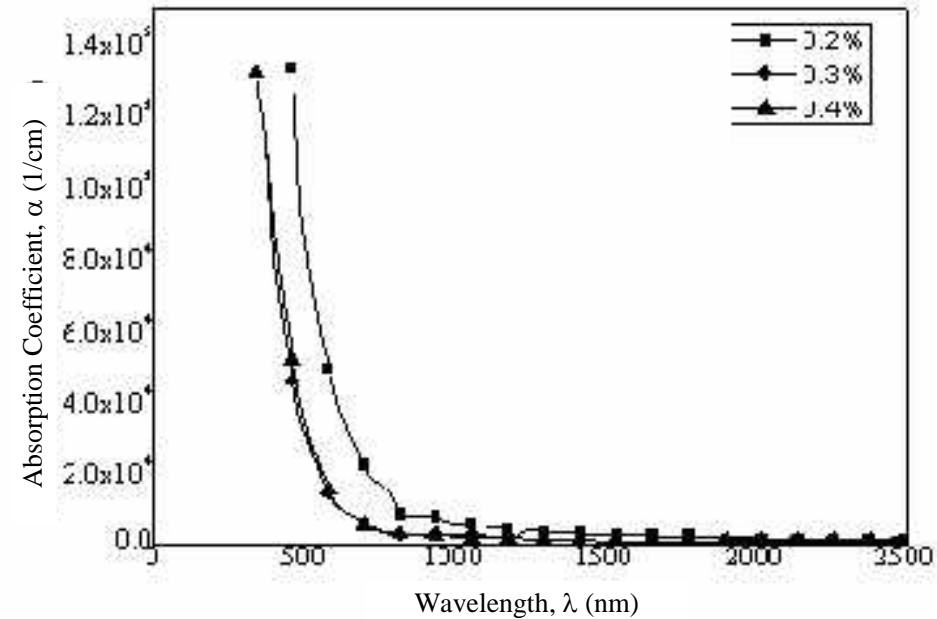

Fig. 3. The absorption coefficient at different indium concentration for $600 \mathrm{~nm}$ GaAs thin film.

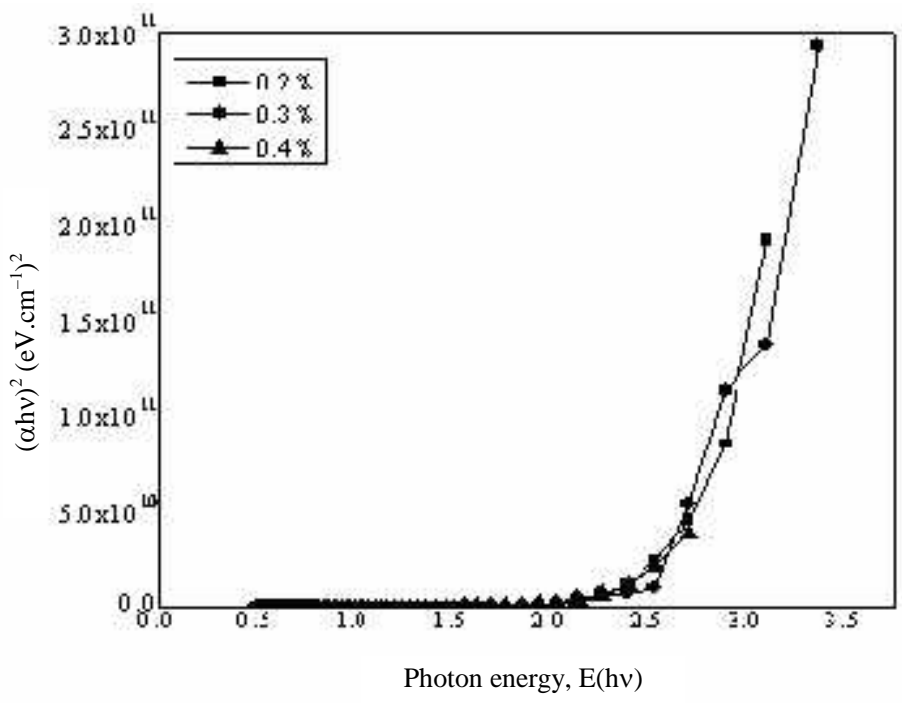

Fig. 4. Variation of $(\alpha h v)^{2}$ with photon energy of indium concentration for $600 \mathrm{~nm}$ GaAs thin film.

Fig. 4 shows the variation of $(a h v)^{2}$ versus photon energy for different indium concentration of GaAs thin films. The band gap can be obtained from extrapolation of the straight line portion of $(\alpha \boldsymbol{h} v)^{2}$ vs $\boldsymbol{h} v$ plot to $\boldsymbol{h v}=\mathbf{0}$. The band gap energies found to be $2.88,2.83$ and $2.39 \mathrm{eV}$ for $0.2,0.3$ and $0.4 \%$ indium concentration, respectively. The band gap decreases with increase of doping concentration. The decrease of energy band gap leads to strong disorder when a small amount of Ga atoms are replaced by In. This happens due to the large disparity in the electronegativity and the atomic size 
between in and $\mathrm{Ga}$ in $\mathrm{In}_{\mathrm{x}} \mathrm{Ga}_{1-\mathrm{x}} \mathrm{As}$. The indium atom brings several perturbations in the host crystal (Srivani et al. 2014).

From Fig. 5, the behavior of refractive index with wavelength can be observed. Firstly, they increase gradually. After attaining a maximum peak they decreases abruptly and at higher wavelengths they start to increase again. The sharp decrease depends on the surface and volume imperfections. Low refractive index occurs due to successive internal reflections or due to the trapped photon energy within the grain boundary (Ong et al. 2000).

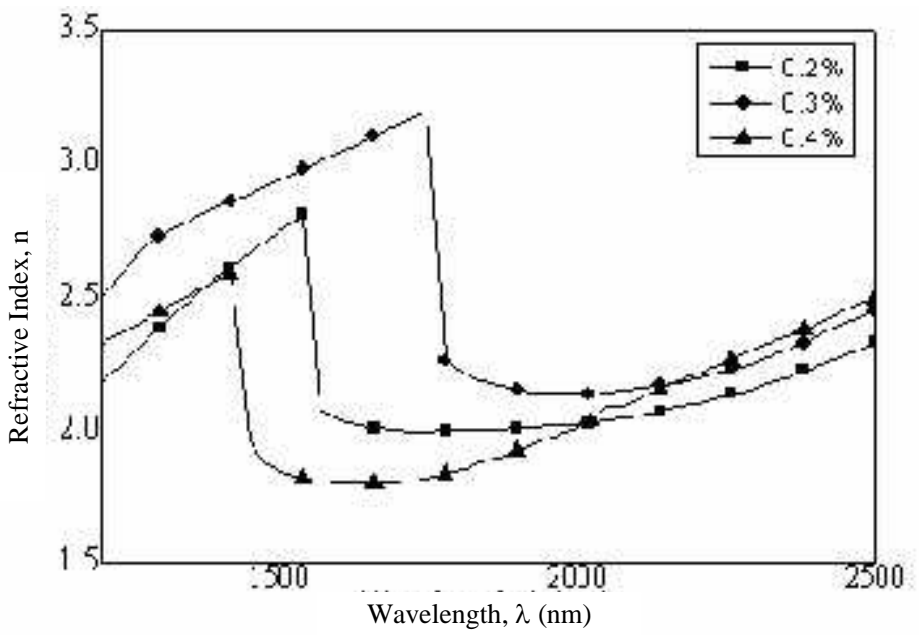

Fig. 5. The reflective index at different indium concentration for $600 \mathrm{~nm} \mathrm{GaAs}$ thin film.

Fig. 6 shows the variation of extinction co-efficient of the films with wavelength for indium concentration $0.2,0.3$ and $0.4 \%$ at a fixed thickness of $600 \mathrm{~nm} \mathrm{GaAs}$ thin films. It was observed that extinction co-efficient decreased with the increase of wavelength.

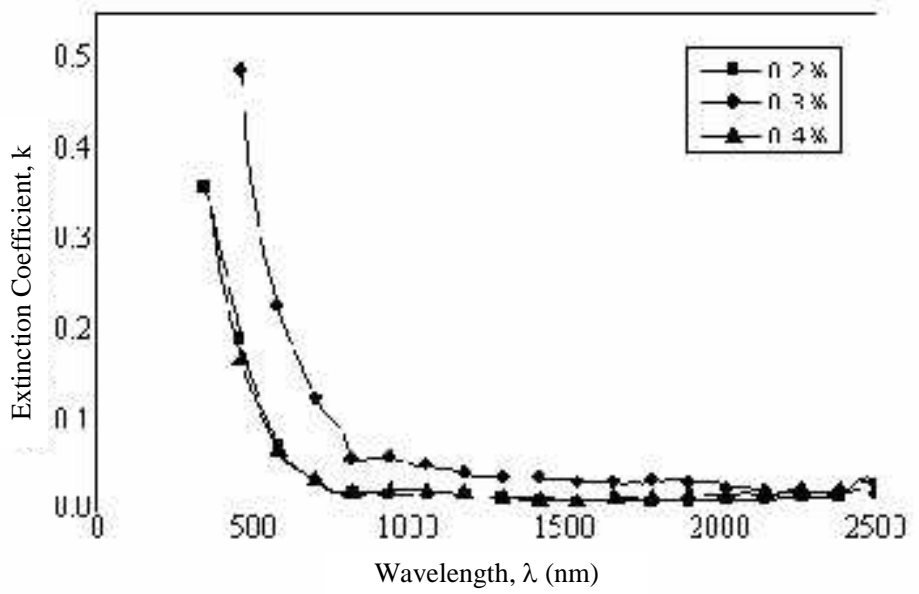

Fig. 6. The extinction co-efficient at different indium concentration for $600 \mathrm{~nm}$ GaAs thin film. 
The changes occurred due to the variation of absorbance. The fall in the extinction co-efficient may be due to the absorption of light at the grain boundaries (Das et al. 2013). The low value of extinction co-efficient is a qualitative indication of excellent surface smoothness of the thin films (Bhaskar et al. 2001). The higher values of extinction co-efficient are the representation of greater attenuation of light in a thin film and also the higher probability of raising the electron transfer across the mobility gap of photon energy.

\section{CONCLUSIONS}

From the present work it can be summarized that, with the increase of doping concentration of indium the transmittance decreases. Absorbance of $0.2 \%$ indium concentration has higher value than the other two percentages concentration. The optical band gap decreases with increasing doping concentration. The refractive index increases with increase of doping concentration. Finally, extinction co-efficient of $0.3 \%$ concentration shows higher values rather than 0.2 and $0.4 \%$ indium doping concentration.

\section{ACKNOWLEDGEMENTS}

The authors are thankful to Experimental Physics Division, Atomic Energy Center, Dhaka and BUET for necessary supports and fruitful discussions.

\section{REFERENCES}

Balkanski, M. and R. F. Wallis. 2000. Semiconductor Physics and Applications. Oxford University Press.

Biswas, M., M. Sharmin, C. Das, J. Podder and S. Choudhury. 2016. Structural and optical characterization of magnesium doped zinc oxide thin films deposited by spray pyrolysis. Dhaka Univ. J. Sci. 64(1): 1-6.

Chowdhury, F. R., S. Chudhury, F. Hasan and T. Begum. 2011. Optical properties of undoped and indium-doped tin oxide thin films. J. Bangladesh Acad. Sci. 35(1): 99-111.

Das, C., J. Begum, T. Begum and S. Choudhury. 2013. Effect of thickness on the optical properties of GaAs thin films. J. Bangladesh Acad. Sci. 37(1): 83-91.

Halliday, D. P., J. M. Eggleston, K. Y. Lee, J. E. F. Frost and S. P. Beaumant. 1995. Optical properties of ultrathin $50 \mathrm{~nm}$ GaAs membranes. Solid State Commun. 96(6): 359-365.

Heavans, O. S. 1965. Optical properties of thin Solid films. Butterworths Scientific Publications, London.

Majeed, A. H. 2011. Effect of Rate of deposition on the optical parameters of GaAs films. Eur. J. Sci. Res. 57(3): 478-484.

Mott, N. F. and E. A. Davis. 1979. Electronics process in non-crystalline materials. Oxford University Press.

Ong, H. C., J. Y. Dai, K. C. Hung, Y. C. Chan and S. T. Ho. 2000. Electronic Structures of Polycrystalline $\mathrm{ZnO}$ thin films probed by electron energy loss spectroscopy. Appl. Phys. Lett. 
77(10): 1484.

Salina, M, R. Ahmad, A. B. Suriani, and M. Rusop, 2012. Bandgap alteration of transparent zinc oxide thin film with Mg Dopant. Trans. Electr. Electron. Mater. 13(2): 64-68.

Sharmin, M., S. Choudhury, N. Akhtar and T. Begum. 2012. Optical and Transport properties of ptype GaAs. J. Bangladesh Acad. Sci. 36(1): 97-108.

Srivani, A., V. R. Murthy and G. V. Raghavaiah. 2014. Research in physical properties of $\mathrm{Al}_{\mathrm{x}} \mathrm{Ga}_{1-\mathrm{x}}$ As III-V arsenide ternary semiconductor alloys. Int. J. Phys. Sci. 9(12): 281-291.

Tauc, J. 1974. Amorphous and liquid semiconductors. Plenum, London. pp.159.

Tomlin, S. G. 1968. Optical reflection and transmission formulae for thin films. J. Phys. D: Appl. Phys. 1: 1667.

Tsuji, S., E. Iri and T. Hideyuki. 1992. Electronic properties of GaAs thin film deposited on silica substrate by RF sputtering. Jpn. J. Appl. Phy. 31(3): 880-881.

Yan-Ping, YAO., L. Chun-Ling, Q. Zhong-Liang, L. Mei, G. Xin and B. Bao-Xue. 2008. Structural, optical and electrical Properties of hydrogen-doped amorphous GaAs thin films. Chin. Phys. Lett. 25(3): 1071.

(Received revised manuscript on 21 September, 2016) 